\title{
Research on Stress Response of Cantilever Beam under Super-Gaussian Base Acceleration Excitation
}

\author{
LIU Yuman ${ }^{1, a}$, JIANG Yu ${ }^{1, b}$, CHEN Xun ${ }^{1, c}$ \\ ${ }^{1}$ Science and Technology on Integrated Logistics Support Laboratory, College of Mechatronics \\ Engineering and Automation, National University of Defense Technology \\ Changsha, 410073, Hunan, P R China \\ a309774797@qq.com, bjiangyunudt@sina.com, chenxunnudt@sina.com
}

\begin{abstract}
Keywords: super-Gaussian, base acceleration excitation, stress response, cantilever beam
\end{abstract}
\begin{abstract}
Stress response is the foundation for calculating a structure's fatigue life during vibration testing. The frequency-domain method based on stress frequency-response function (SFRF) is widely used because of its convenience. For Gaussian random excitation, the frequency-domain method may have a good accuracy. But when it comes to super-Gaussian random excitation, this method is not that applicable. In this paper, the time-domain method for calculating the stress response of a cantilever beam under super-Gaussian random excitation is proposed by using mode superimposition method. Two numerical examples are presented with the help of MATLAB. As a result, for linear system, when the excitation is Gaussian (super-Gaussian) distributed, the stress response is Gaussian (super-Gaussian) distributed. And when the frequency span of the excitation keeps the same, the larger the kurtosis of the excitation is, the larger the kurtosis of the response will be. For super-Gaussian stress response, the bandwidth of the excitation can influence the kurtosis of the stress response. Specifically, the narrower the bandwidth is, the larger the kurtosis will be.
\end{abstract}

\section{Introduction}

Vibration testing is an important job before equipments come into service because vibration is one of the vital reasons that lead them to failure. In laboratory, the testing is typically performed with an electro-dynamic shaker which gives the object base acceleration excitation [1,2]. Until very recently, the random vibration in the environment has been treated as Gaussian random process [3]. But in practice, many observations have confirmed that the random excitation do not follow Gaussian distribution in some cases. There may be distinct difference between the stress response when a system subjected to Gaussian and super-Gaussian excitation though they have the same PSD [4]. If the super-Gaussianity of the excitation is ignored, stress response may be inaccurate, thus leading incorrect result in fatigue life prediction.

Grigoriu [5] investigates the problems for linear system under non-Gaussian $\alpha$ stable process in the abstract. But it is not applicable for structure response under non-Gaussian excitation in practice. G.Q.CAI [6] proposes a approach of nonlinear filter which offered a versatile way to model non-Gaussian stochastic processes with various shapes of the probability density and the spectral density. Numerical examples show that the proposed procedure has much higher accuracy than the conventional linearization in cases of strong system nonlinearity or high excitation non-Gaussianity.

Iyengar and Jaiswal [7] investigates the response of linear system under non-Gaussian excitation by transforming the non-Gaussian process into several Gaussian polynomials. Cheng [8] compares the differences in the response corresponding to Gaussian, steady non-Gaussian and burst non-Gaussian base excitations with identical PSD in terms of RMS and kurtosis.

Although researches about the response of system under non-Gaussian excitation have been presented, how the properties of the excitation influence the non-Gaussianity of the stress response is not clear. In this paper, a time-domain method for calculating the stress response of a cantilever beam under super-Gaussian base acceleration excitation is proposed. Based on the formula derived, two numerical examples are presented. As a result, the factors influencing the non-Gaussianity of the stress response are determined: the kurtosis and the bandwidth of the excitation. 


\section{Super-Gaussian random process}

Non-Gaussian random process is relative to Gaussian random process. For ergodic process, Gaussian process X(t) means its probability density function (pdf) is Gaussian distributed:

$$
f(x)=\frac{1}{\sqrt{2 \pi \sigma}} \exp \left[-\frac{(x-\mu)}{2 \sigma^{2}}\right] .
$$

where $\mu$ is the mean value and $\sigma$ is the standard deviation.

Generally, random process whose pdf is not Gaussian distributed can be treated as non-Gaussian process. Given a non-Gaussian process $\mathrm{Z}(\mathrm{t})$, the degree of its non-Gaussianity is quantified by skewness $\gamma_{3}$ and kurtosis $\gamma_{4}$ [9], defined as:

$$
\left\{\begin{array}{l}
\gamma_{3}=\frac{m_{3}}{\sigma_{Z}^{3}}=\frac{E\left[Z^{3}(t)\right]}{\sigma_{Z}^{3}} \\
\gamma_{4}=\frac{m_{4}}{\sigma_{Z}^{4}}=\frac{E\left[Z^{4}(t)\right]}{\sigma_{Z}^{4}}
\end{array} .\right.
$$

For Gaussian process, $\gamma_{3}=0$ and $\gamma_{4}=3$. For non-Gaussian process, $\gamma_{3} \neq 0$ or $\gamma_{4} \neq 3$. According to whether $\gamma_{3}=0$ or not, non-Gaussian process can be divided into symmetrical non-Gaussian and skewed non-Gaussian. According to whether $\gamma_{4}>3$ or not, non-Gaussian can be divided into super-Gaussian and sub-Gaussian. What we investigated in this paper is symmetrical super-Gaussian.

In time-domain, super-Gaussian has some different characteristics from Gaussian process. As shown in Fig. 1(b), there are high-excursion distributed over time. A comparison between standardized Gaussian and super-Gaussian with the same PSD is shown in Fig. 1.

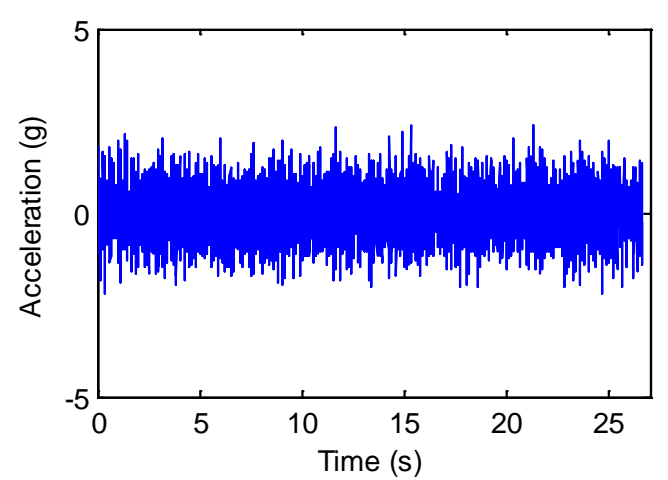

(a)

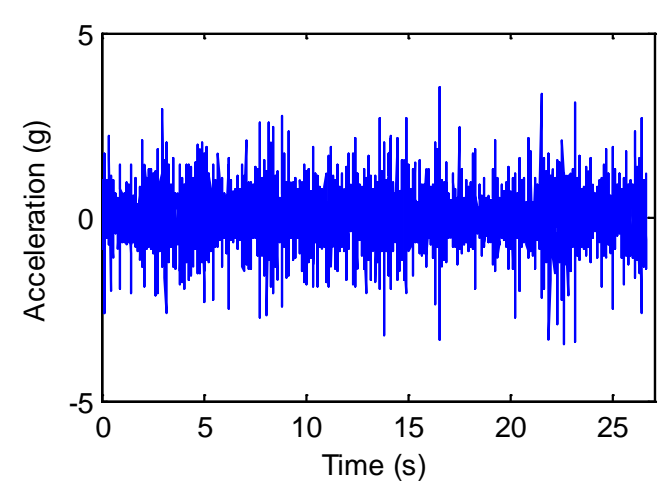

(b)

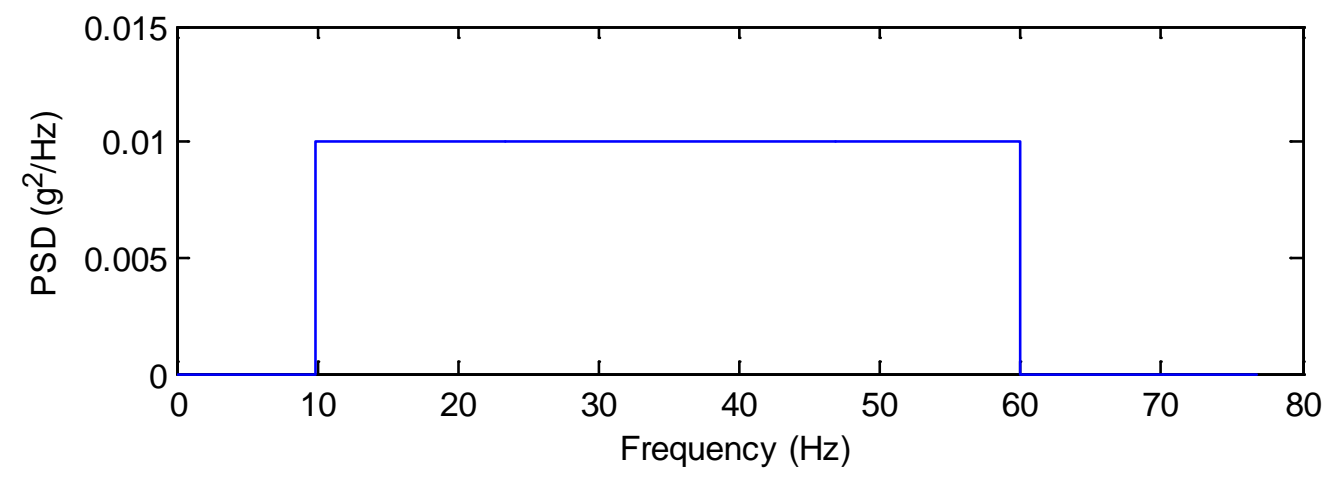

(c)

Fig.1 Gaussian and super-Gaussian random process with the same PSD: (a) Gaussian; (b) super-Gaussian, $\gamma_{4}=5$; (c) PSD 


\section{Theoretical background of vibration}

Base acceleration excitation is the most common practice for vibration testing in laboratory. Fig. 2 shows the movement of a cantilever beam with denoted acceleration excitation at the base tip.

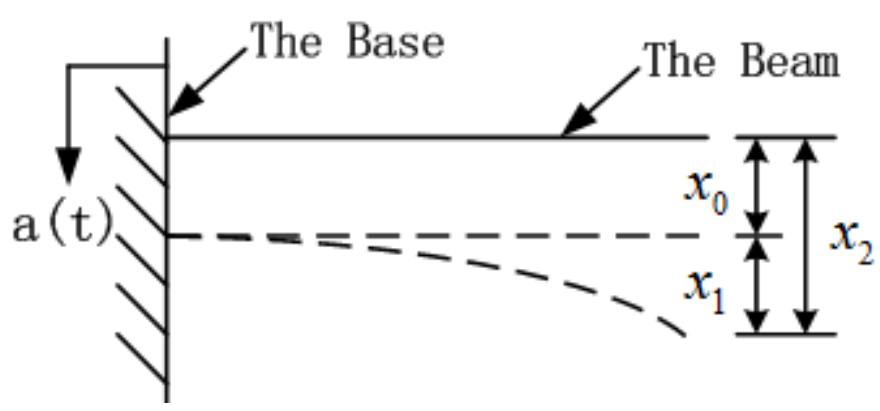

Fig.2 Base excitation of a cantilever beam

Where $x_{2}, x_{1}$ and $x_{0}$ are the absolute displacement of the beam, the displacement of the base, and the relative displacement between the beam and the base respectively, which satisfy the equation below

$$
x_{0}+x_{1}=x_{2} \text {. }
$$

When we take a piece of beam as the subject investigated, the differential equation of motion can be given by

$$
d m \ddot{x}_{2}+c \dot{x}_{1}+k x_{1}=0 .
$$

where $d m, c$ and $k$ are, respectively the mass, damping and stiffness of the differential element.

Substituting Eq. 3 into Eq. 4, results in:

$$
d m \ddot{x}_{1}+c \dot{x}_{1}+k x_{1}=-d m \ddot{x}_{0} .
$$

For constant-section cantilever beam, $d m$ can be denoted as $d m=\rho$ Ads. Where $\rho$ is the density of the material, $A$ is the area of cross section, and $d s$ is the length of differential element. So the right side of Eq. 5 can be treated as inertial force over time which depends on the base acceleration:

$$
f=-\rho A a(t) d s \text {. }
$$

For unit length, the distributed force can be written as

$$
f(t)=-\rho A a(t) \text {. }
$$

Usually in stress response analysis, the motion relative to the base is of interest. According to the analysis above, base acceleration excitation is equivalent to the distributed force shown in Fig. 3.

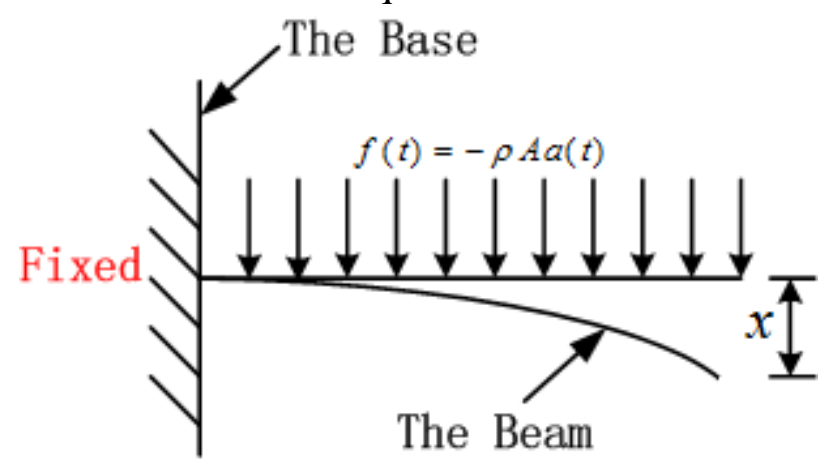

Fig.3 Equivalent system to that shown in Fig. 2 


\section{Dynamic stress analysis}

The subject investigated in this paper is Euler-Bernoulli beam. Stress is proportional to the bending moment, which has a relationship as follows:

$$
\sigma(s, t)=\frac{M(s, t)}{W} .
$$

where $M$ and $W$ are bending moment and bending modulus of a certain cross-section. $s$ is the distance from the cross-section to the fixed end. For Euler-Bernoulli beam, the bending moment is relative to the deflection [10], which can be expressed as follows:

$$
M(s, t)=E I \frac{\partial^{2} x(t)}{\partial s^{2}} .
$$

where $E$ is the elastic modulus and $I$ is the moment inertia of the cross-section.

According to mode superposition method and Galerkin method [11], the deflection can be expressed as

$$
x(s, t)=\sum_{i=1}^{n} \varphi_{i}(s) q_{i}(t) .
$$

where $\varphi_{i}(s)$ is $i$-th mode of cantilever beam, $q_{i}(t)$ is the generalized coordinate of $i$-th mode.

$$
\varphi_{i}(s)=\sin \beta_{i} s-\sinh \beta_{i} s-\alpha_{i}\left(\cos \beta_{i} s-\cosh \beta_{i} s\right) .
$$

where $\beta_{i} l=C_{i}$ is a constant for a specified $i$, and $l$ is the length of the beam. $\alpha_{i}=\frac{\sin \beta_{i} l+\sinh \beta_{i} l}{\cos \beta_{i} l+\cosh \beta_{i} l}$.

In order to get the expression of $q_{i}(t)$, Lagrange equation is a good choice to use.

First, the velocity of the cross-section can be written as

$$
v(s, t)=\frac{\partial x(s, t)}{\partial t}=\sum_{i=1}^{n} \varphi_{i}(s) \dot{q}_{i}(t) .
$$

So the kinetic energy of the beam is

$$
\begin{aligned}
T & =\frac{1}{2} \int_{0}^{l} \rho A v^{2}(s, t) d s=\frac{1}{2} \int_{0}^{l} \rho A\left[\sum_{i=1}^{n} \varphi_{i}(s) \dot{q}_{i}(t)\right]\left[\sum_{j=1}^{n} \varphi_{j}(s) \dot{q}_{j}(t)\right] d s \\
& =\frac{1}{2} \sum_{i=1}^{n} \sum_{j=1}^{n} \dot{q}_{i}(t) \dot{q}_{j}(t) \int_{0}^{l} \rho A \varphi_{i}(s) \varphi_{j}(s) d s=\frac{1}{2} \sum_{i=1}^{n} M_{i} \dot{q}_{i}^{2}(t) .
\end{aligned}
$$

where $M_{i}=\int_{0}^{l} \rho A \varphi_{i}^{2}(s) d s$,can be defined as the generalized mass for $q_{i}(t)$. During the simplification, the orthogonality of each mode is used:

$$
\int_{0}^{l} \varphi_{i}(s) \varphi_{j}(s) d s=\left\{\begin{array}{r}
\int_{0}^{l} \varphi_{i}^{2}(s) d s, i=j \\
0, i \neq j
\end{array} .\right.
$$

According to Eq. 9 and Eq. 10, bending moment at each cross-section can be written as

$$
M(s, t)=E I \frac{\partial^{2} x(t)}{\partial s^{2}}=E I \sum_{i=1}^{n} \ddot{\varphi}_{i}(s) q_{i}(t) .
$$

So the elastic potential energy of the beam is 


$$
\begin{aligned}
U & =\frac{1}{2} \int_{0}^{l} \frac{M^{2}(s, t)}{2 E I} d s=\frac{1}{2} \int_{0}^{l} E I\left[\sum_{i=1}^{n} \ddot{\varphi}_{i}(s) q_{i}(t)\right]\left[\sum_{j=1}^{n} \ddot{\varphi}_{j}(s) q_{j}(t)\right] d s \\
& =\frac{1}{2} \sum_{i=1}^{n} \sum_{j=1}^{n} q_{i}(t) q_{j}(t) \int_{0}^{l} E I \ddot{\varphi}_{i}(s) \ddot{\varphi}_{j}(s) d s=\frac{1}{2} \sum_{i=1}^{n} K_{i} q_{i}^{2}(t) .
\end{aligned}
$$

where $K_{i}=\int_{0}^{l} E I \ddot{\varphi}_{i}^{2}(s) d s$, can be defined as the generalized stiffness for $q_{i}(t)$. And $K_{i}=\omega_{i}^{2} M_{i}, \omega_{i}$ is $i$-th resonant frequency.

The virtual displacement can be written as

$$
\delta x(s, t)=\sum_{i=1}^{n} \varphi_{i}(s) \delta q_{i}(t) .
$$

So the virtual work the distributed force $f(t)$ does can be expressed as

$$
\delta W=\int_{0}^{l} f(t)\left[\sum_{i=1}^{n} \varphi_{i}(s) \delta q_{i}(t)\right] d s=\sum_{i=1}^{n} Q_{i}(t) \delta q_{i}(t) .
$$

In Eq. 18, generalized force is defined:

$$
Q_{i}(t)=\int_{0}^{l} f(t) \varphi_{i}(s) d s
$$

Substituting Eq. 13, Eq. 16 and Eq. 19 into Lagrange equation

$$
\frac{d}{d t}\left(\frac{\partial T}{\partial \dot{q}_{i}}\right)-\frac{\partial T}{\partial q_{i}}+\frac{\partial U}{\partial q_{i}}=Q_{i}
$$

results in

$$
\ddot{q}_{i}(t)+\omega_{i} q_{i}(t)=\frac{Q_{i}}{M_{i}} .
$$

Essentially, Eq. 21 can be treated as the motion equation of non-damped single degree of freedom system. With the help of Duhamel integral, the solution of $q_{i}(t)$ is

$$
q_{i}(t)=A_{i} \cos \omega_{i} t+B_{i} \sin \omega_{i} t+\frac{1}{M_{i} \omega_{i}} \int_{0}^{t} Q_{i}(\tau) \sin \omega_{i}(t-\tau) d \tau
$$

The first two items is transient response, and the third item is stable response. Neglecting the transient response driven by the initial condition, Eq. 22 can be rewritten as

$$
q_{i}(t)=\frac{1}{M_{i} \omega_{i}} \int_{0}^{t} Q_{i}(\tau) \sin \omega_{i}(t-\tau) d \tau
$$

Actually, damp can not be ignored. So taking damping ratio $\xi_{i}$ into consideration, Eq. 23 becomes

$$
q_{i}(t)=\frac{1}{M_{i} \omega_{i}} \int_{0}^{t} Q_{i}(\tau) e^{-\xi_{i} \omega_{i}(t-\tau)} \sin \omega_{i}^{d}(t-\tau) d \tau
$$

where $\omega_{i}^{d}=\omega_{i} \sqrt{1-\xi_{i}^{2}}$.

By substituting Eq. 7, Eq. 19 and Eq. 24 into Eq. 10, the dynamic deflection can be expressed as follows: 


$$
x(s, t)=\sum_{i=1}^{n} \frac{\varphi_{i}(s)}{\omega_{i}^{d} \int_{0}^{l} \varphi_{i}^{2}(s) d s} \int_{0}^{t}\left[\int_{0}^{l} \varphi_{i}(s)\right] a(\tau) e^{-\xi_{i} \omega_{i}(t-\tau)} \sin \omega_{i}^{d}(t-\tau) d \tau .
$$

Substituting Eq. 9 and Eq. 25 into Eq. 8, the dynamic stress response becomes

$$
\sigma(s, t)=\frac{E I}{W} \sum_{i=1}^{n} \frac{\partial^{2} \varphi_{i}(s)}{\partial s^{2}} \frac{1}{\omega_{i}^{d} \int_{0}^{l} \varphi_{i}^{2}(s) d s} \int_{0}^{t}\left[\int_{0}^{l} \varphi_{i}(s)\right] a(\tau) e^{-\xi_{i} \omega_{i}(t-\tau)} \sin \omega_{i}^{d}(t-\tau) d \tau .
$$

\section{Numerical examples}

The subject investigated is a cantilever beam made of Al 6061-T6, whose mechanical properties are shown in Table 1 . The dimensions are $\mathrm{l}=200 \mathrm{~mm}, \mathrm{~b}=20 \mathrm{~mm}, \mathrm{~h}=2 \mathrm{~mm}$.

Table 1.The mechanical properties of Al 6061-T6

\begin{tabular}{|c|c|c|c|}
\hline Elastic modulus[GPa] & Poisson ratio & Ultimate stress[MPa] & Density $\left[\mathrm{kg} / \mathrm{m}^{3}\right]$ \\
\hline 69 & 0.33 & 275 & 2700 \\
\hline
\end{tabular}

Usually, taking the first four modes into account may have a satisfying accuracy. With the help of finite element analysis software ANSYS, the first four modal frequency are $\omega_{1}=41.23 \mathrm{~Hz}, \omega_{2}=258.76 \mathrm{~Hz}, \omega_{3}=396.55 \mathrm{~Hz}, \omega_{4}=727.62 \mathrm{~Hz}$.According to [11] , the parameters in Eq. 11 can be calculated. The results are $\beta_{1}=9.375, \beta_{2}=23.47, \beta_{3}=39.27, \beta_{4}=54.98$, $\alpha_{1}=1.362, \quad \alpha_{2}=0.982, \alpha_{3}=1.001, \alpha_{4}=1.000$. The damp ratio is assumed to be $\xi_{i}=0.02$.

\section{Example.1.}

The super-Gaussianity of the excitation may influence that of stress response. The properties of the base acceleration excitation in example 1 are shown in Table $2.10-60 \mathrm{~Hz}$ covers the first modal frequency. $100-200 \mathrm{~Hz}$ does not cover any modal frequency and $10-800 \mathrm{~Hz}$ covers the first four modal frequencies. According to the properties in frequency-domain, each of the random signal in time-domain is simulated, which is shown in Fig. 1. Substituting the random signal into Eq. 26, the dynamic stress response at each point of the cantilever beam can be acquired, as well as the non-Gaussianity. The results of example 1 are shown in Fig. 4.

Table 2. The properties of the base acceleration in example 1

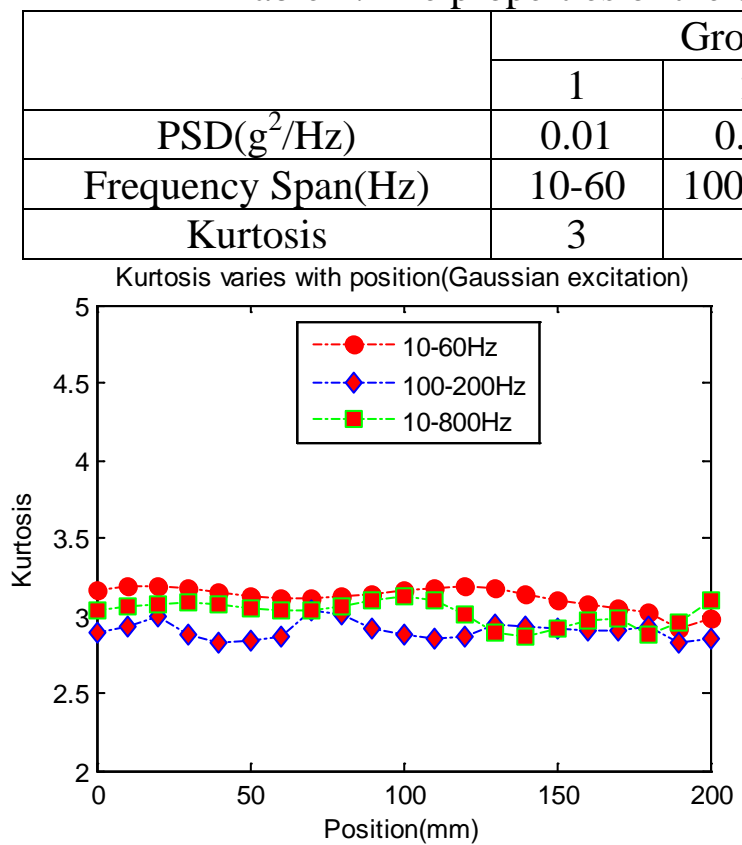

(a)

\begin{tabular}{|l|c|c|c|c|}
\hline 2 & \multicolumn{3}{|c|}{ Group 2 } \\
\hline 2 & 3 & 1 & 2 & 3 \\
\hline 3 & 0.01 & 0.01 & 0.01 & 0.01 \\
\hline-200 & $10-800$ & $10-60$ & $10-60$ & $10-60$ \\
\hline
\end{tabular}

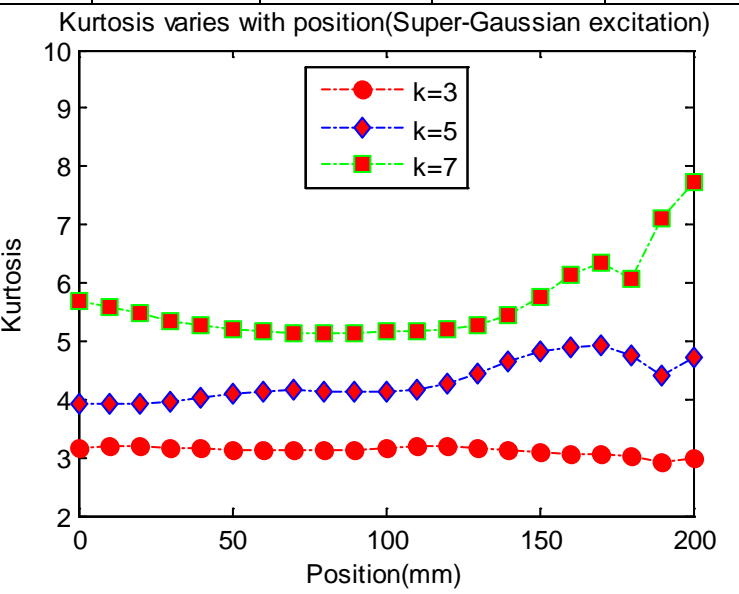

(b)

Fig.4 The kurtosis of stress response varies with position. (a) Gaussian excitation (b) non-Gaussian excitation 
From Fig. 4(a), we can see if the base acceleration excitation is Gaussian distributed, no matter what the frequency span is, the kurtosis at any point of the cantilever beam almost equals to 3. So we have good reason to believe the stress response is Gaussian distributed. From Fig. 4(b), the same result reoccurs when $k=3$. But when $k$ is greater than 3 , such as 5 or 7 , the kurtosis of the stress response is greater than 3 , too. Besides, when the frequency span of the base excitation keeps the same, the larger the kurtosis of excitation is, the larger the kurtosis of the stress response will be.

Example.2.

Table 3. The properties of the base acceleration in example 2

\begin{tabular}{|c|c|c|c|}
\hline & 1 & 2 & 3 \\
\hline PSD $\left(\mathrm{g}^{2} / \mathrm{Hz}\right)$ & 0.01 & 0.01 & 0.01 \\
\hline Frequency Span(Hz) & $10-30$ & $10-60$ & $10-100$ \\
\hline Kurtosis & 5 & 5 & 5 \\
\hline
\end{tabular}

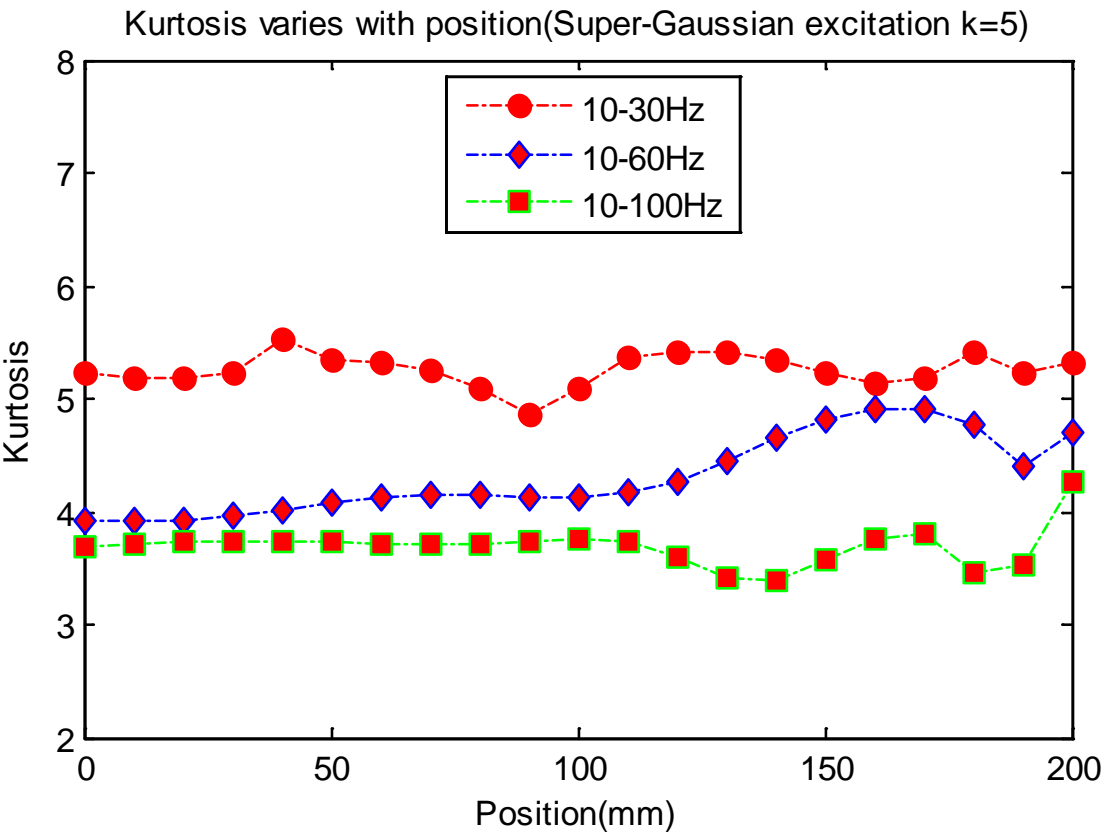

Fig.5 The kurtosis of stress response varies with position.

From Fig. 5, we can see when kurtosis of the base excitation is greater than 3, the kurtosis of the stress response is greater than 3 , too, which validates the conclusion in example.1. In addition, when the kurtosis of the excitation keeps the same, the bandwidth of the excitation can influence the super-Gaussianity of the stress response. To be specific, the narrower the bandwidth is, the larger the kurtosis of the stress response will be.

\section{Summary}

Super-Gaussian random process widely exists all around the actual environment, the stress response of which is of great importance for fatigue life predicting. In this paper, a time-domain method for calculating the stress response of cantilever beam under super-Gaussian base acceleration excitation is proposed with the help of mode superposition method and Lagrange equation. Based on the method, two numerical examples are conducted in the end. According to the results in the study, the following conclusions can be drawn. For linear system:

1). The super-Gaussianity of the excitation can influence the super-Gaussianity of the stress response. To be specific, if the excitation is Gaussian (super-Gaussian) distributed, the stress response is Gaussian (super-Gaussian) distributed, too.

2). For super-Gaussian excitation, the kurtosis of the excitation can influence the kurtosis of the stress response. In detail, when the frequency span of the excitation keeps the same, the larger the kurtosis of the excitation is, the larger the kurtosis of the stress response will be. 
3). For super-Gaussian excitation, the bandwidth of the excitation can influence the kurtosis of the stress response. Specifically, the narrower the bandwidth is, the larger the kurtosis of the stress response will be.

\section{References}

[1] M. Razeto, J. C. Golinval and M. Geradin:12th International Modal Analysis Conference, Honolulu, Hawaii, 1994.

[2] M.A. Blair: 11th International Modal Analysis Conference, Kissimmee, Florida, 1993.

[3] Cho. W. Solomon To: submitted to Probabilistic Engineering Mechanics (2012)

[4] A. Steinwolf: submitted to Probabilistic Engineering Mechanics (1999)

[5] M. Grigoriu: submitted to Probabilistic Engineering Mechanics (1995)

[6] G.Q. Cai and Y. Suzuki: submitted to Nonlinear Dynamics (2005)

[7] N.R. Iyengar and O.R. Jaiswal: submitted to Probabilistic Engineering Mechanics (1993)

[8] Hongwei Cheng and Junyong Tao: submitted to Journal of Vibroengineering (2014)

[9] R. Sheldon: Stochastic Processes.(John Wiley \& Sons, Inc, New York 1996).

[10] Qingtan Liu: Mechanics of Meterials.(China Machine Press, Beijing 2004).

[11] S.R. Singiresu: Mechanical Vibrations.(Tsinghua University Press, Beijing 2009). 